Abstract submitted to the Microscopy \& Microanalysis 2009 Conference

\title{
Cation Stoichiometry from Microanalysis of Complex Perovskite Oxides
}

\author{
S. Kolesnik, B. Dabrowski, and J. Mais, \\ Department of Physics, Northern Illinois University, DeKalb, IL 60115, U.S.A.
}

The solid state synthesis method of polycrystalline complex perovskite oxides requires high temperature annealing, during which the sample stoichiometry should be preserved. In this study, we investigate polycrystalline samples of $\mathrm{SrMn}_{1-\mathrm{x}} \mathrm{Ru}_{\mathrm{x}} \mathrm{O}_{3}$ [1] and $\mathrm{La}_{1-3 \mathrm{x}} \mathrm{Ba}_{3 \mathrm{x}} \mathrm{Mn}_{1-\mathrm{x}} \mathrm{Mo}_{\mathrm{x}} \mathrm{O}_{3}$. These particular materials are interesting from the point of view of possible volatilization of $\mathrm{Ru}$ and Mo, respectively.

We have verified the composition of the synthesized materials. The cation ratio in this material was determined by energy dispersive x-ray spectroscopy (EDXS) analysis in a Hitachi S-4700-II scanning electron microscope. Typically, 5-15 spot spectra were collected across the surface of sintered pellets. The spectra were taken automatically at the pre-determined spots using the Multipoint option of the EDXS software (EDAX) (Fig. 1). From the spectra, the atomic percent concentrations of each cation were determined using the standardless ZAF analysis. The sum of all the concentrations was kept to $100 \%$. Oxygen was omitted in the analysis. The effective cation contents $\mathrm{x}_{\mathrm{eff}}$ were determined and compared to the nominal contents in the perovskite formula. In Fig. 2, the results for $\mathrm{SrMn}_{1-\mathrm{x}} \mathrm{Ru}_{\mathrm{x}} \mathrm{O}_{3}$ are presented. We used two different reference cations: either A-site ions [Sr: Fig. 2 (a)] or B-site ions [Mn+Ru: Fig. 2 (b)]. The results of the latter procedure give better results that agree with the expected cation contents, which have also been confirmed by neutron powder diffraction (NPD) [Fig. 2 (b)].

Although in the perovskite formula $\mathrm{ABO}_{3}$ the expected ratio of A-site cations to B-site cations is always equal to 1 , we have noticed systematically different ratios in our $\mathrm{La}_{1-3 \mathrm{x}} \mathrm{Ba}_{3 \mathrm{x}} \mathrm{Mn}_{1-\mathrm{x}} \mathrm{Mo}_{\mathrm{x}} \mathrm{O}_{3}$ samples [Fig. 3 (b)]. We believe that this result reflects the inaccuracy of the standardless ZAF analysis. We have verified this claim by the EDXS analysis of a $\mathrm{La}_{0.5} \mathrm{Ba}_{0.5} \mathrm{MnO}_{3}$ reference sample. The composition of that sample was confirmed by NPD [2]. We have found that the ratio $\mathrm{x}_{\text {eff }}(\mathrm{La}) / \mathrm{x}_{\text {eff }}(\mathrm{Ba})=1.00(2)$ and $\mathrm{x}_{\text {eff }}(\mathrm{Mn}) /\left[\mathrm{x}_{\text {eff }}(\mathrm{La})+\mathrm{x}_{\text {eff }}(\mathrm{Ba})\right]=0.87(2)$ similar as for all the $\mathrm{La}_{1-}$ ${ }_{3 \mathrm{x}} \mathrm{Ba}_{3 \mathrm{x}} \mathrm{Mn}_{1-\mathrm{x}} \mathrm{Mo}_{\mathrm{x}} \mathrm{O}_{3}$ samples. To account for that, we have divided the atomic percent concentrations of each cation in $\mathrm{La}_{1-3 x} \mathrm{Ba}_{3 x} \mathrm{Mn}_{1-x} \mathrm{Mo}_{\mathrm{x}} \mathrm{O}_{3}$ by the sum of A-site cation concentrations $(\mathrm{La}+\mathrm{Ba})$. The results are presented in Fig. 3.

Our EDXS results on polycrystalline perovskite oxides show that the determined effective cation contents usually agree with the nominal values. No $\mathrm{Ru}$ deficiency in $\mathrm{SrMn}_{1-\mathrm{x}} \mathrm{Ru}_{\mathrm{x}} \mathrm{O}_{3}$ or Mo deficiency in $\mathrm{La}_{1-3 \mathrm{x}} \mathrm{Ba}_{3 \mathrm{x}} \mathrm{Mn}_{1-\mathrm{x}} \mathrm{Mo}_{\mathrm{x}} \mathrm{O}_{3}$ has been noticed.

[1] S. Kolesnik, B. Dabrowski, and O. Chmaissem, Phys. Rev. B 78, 214425 (2008).

[2] O. Chmaissem, B. Dabrowski, S. Kolesnik, J. Mais, J. D. Jorgensen, S. Short, C. E. Botez, and P.W. Stephens, Phys. Rev. B 72, 104426 (2005).

[3] Results shown here are derived from work at Argonne National Laboratory, which is operated by the U. of Chicago for the U. S. Department of Energy under contract DE-AC0206CH11357. Work at NIU was supported by the NSF grant no. DMR-0706610. S. K. would like to thank Russell E. Cook and Jon Hiller for their technical support. 
Abstract submitted to the Microscopy \& Microanalysis 2009 Conference
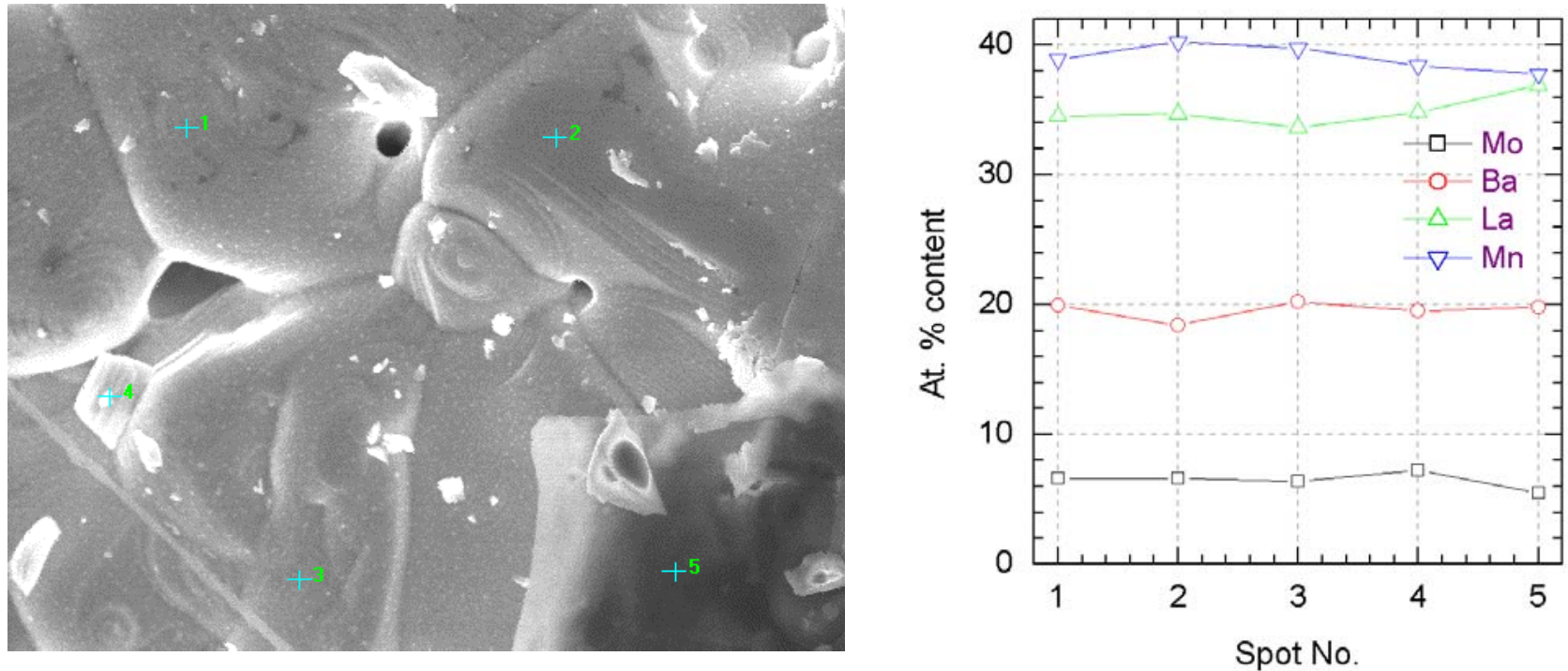

Fig. 1. (left) Typical SEM view of the surface of a sintered pellet. The numbered crosses mark the spots, of which EDXS spectra were taken. (right) At. \% contents of all the cations in the studied sample $\left(\mathrm{La}_{0.64} \mathrm{Ba}_{0.36} \mathrm{Mn}_{0.88} \mathrm{Mo}_{0.12} \mathrm{O}_{3}\right)$.

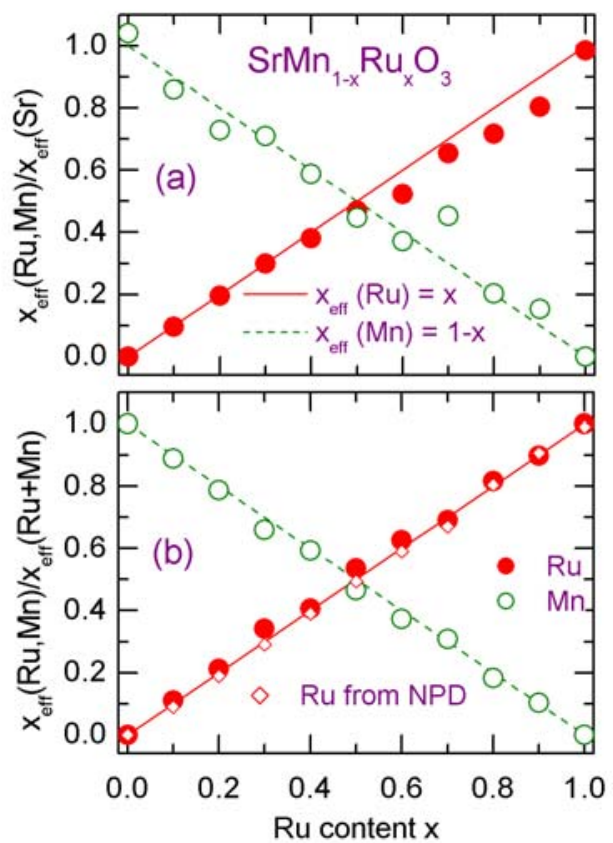

Fig. 2. The effective cation contents for $\mathrm{SrMn}_{1-\mathrm{x}} \mathrm{Ru}_{\mathrm{x}} \mathrm{O}_{3}$ determined from the EDXS analysis (a) A-site cation ( $\mathrm{Sr}$ ) or (b) B-site cations $(\mathrm{Sr}+\mathrm{Ru})$ were used as a reference.

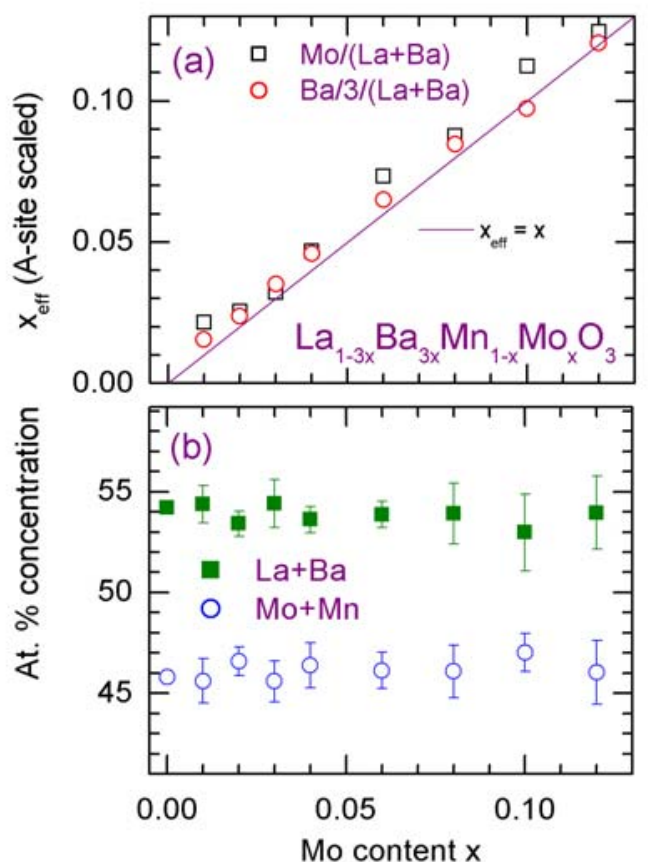

Fig. 3. The effective cation contents in $\mathrm{La}_{1-3 \mathrm{x}} \mathrm{Ba}_{3 \mathrm{x}} \mathrm{Mn}_{1-\mathrm{x}} \mathrm{Mo}_{\mathrm{x}} \mathrm{O}_{3}$ determined from the EDXS analysis by using Asite cations $(\mathrm{La}+\mathrm{Ba})$ as a reference. 